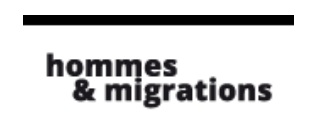

\section{Hommes \& migrations}

Revue française de référence sur les dynamiques

migratoires

\section{8 | 2014}

Les Paris des migrants

\title{
Une cathédrale russe au pied de la tour Eiffel
}

Entretien avec Tatiana Kastouéva-Jean, responsable du Centre RussieNEI à l'Institut français des relations internationales (Ifri).

\section{Marie Poinsot}

\section{(2) OpenEdition}

Journals

Édition électronique

URL : http://journals.openedition.org/hommesmigrations/2998

DOI : 10.4000/hommesmigrations.2998

ISSN : 2262-3353

\section{Éditeur}

Musée national de l'histoire de l'immigration

\section{Édition imprimée}

Date de publication : 1 octobre 2014

Pagination : $67-73$

ISBN : 978-2-919040-29-2

ISSN : $1142-852 X$

\section{Référence électronique}

Marie Poinsot, « Une cathédrale russe au pied de la tour Eiffel », Hommes \& migrations [En ligne], 1308 | 2014, mis en ligne le 01 octobre 2017, consulté le 22 avril 2019. URL : http://

journals.openedition.org/hommesmigrations/2998; DOI : 10.4000/hommesmigrations.2998 


\section{UNE CATHÉDRALE RUSSE AU PIED DE LA TOUR EIFFEL}

Entretien avec TATIANA KASTOUÉVA-JEAN, responsable du Centre Russie-NEI à l'Institut français des relations internationales (Ifri), réalisé par MARIE POINSOT.

\section{Hommes \& Migrations : La présence des immi- grés russes à Paris est ancienne. Pouvez-vous nous rappeler les grandes étapes et les profils sociologiques de cette immigration?}

Tatiana Kastouéva-Jean : La France a été la terre d'asile privilégiée des immigrés russes à plusieurs reprises. On peut parler de trois ou quatre vagues d'émigration russe, d'ampleur inégale et de profils sociologiques très différents.

Dès le début du XIX siècle, un embryon de colonie russe se forme à Paris grâce aux nobles qui y viennent se divertir quelques mois. Cette naissance de la "Russie hors frontière" à Paris a été facilitée par la maîtrise du français de la noblesse russe, qui parfois le parlait mieux que sa langue maternelle ! Vers 1840 arrivent les premiers émigrés politiques qui fuient le règne du tsar autocrate Nicolas ${ }^{\text {er }}$. Leur nombre va grossir au fil des années suivantes. Parmi ces émigrés se distinguent quelques grands philosophes et écrivains comme Mikhail Bakounine, Alexandre Herzen, Ivan Tourgueniev. Le nombre de Russes installés en France vers le milieu du XIX siècle est évalué à plus de 9000 personnes.

Sous Alexandre II, le flux des émigrants et des voyageurs russes à Paris ne tarit pas : on trouve parmi eux des artistes connus, des étudiants, des journa- listes, des militaires, de hauts fonctionnaires et des personnes de l'entourage impérial. De cette époque datent la construction de la cathédrale orthodoxe Saint-Alexandre-Nevsky de la rue Daru (18591861) et la fondation d'une bibliothèque (1874) à l'initiative des étudiants russes de Paris avec l'aide de Tourgueniev dont elle porte toujours le nom. Des liens culturels très forts se tissent à ce moment-là ; l'attirance et l'admiration réciproques sont indéniables. Cela prépare les bases de la grande Alliance franco-russe conclue en 1891 entre l'empereur Alexandre III et le président Sadi Carnot. Cette amitié franco-russe est symbolisée par le pont Alexandre-III inauguré pour l'Exposition universelle de Paris en 1900.

L'une des conséquences imprévues de l'Alliance franco-russe est le choix de la France comme pays d'accueil privilégié par l'émigration russe qui fuit le pays à cause de la Révolution de 1917 et de la guerre civile. C'est la deuxième vague d'émigrés politiques russes, qui dépasse de loin la première par son ampleur. Les chiffres sont difficiles à établir avec exactitude en raison des différents statuts des réfugiés, mais ils seraient plus de 70000 vers le milieu des années 1920. Pour certains, la France n'est qu'un pays de transit vers d'autres destinations. On trouve parmi ces émigrés des aristocrates, 
des proches de la famille impériale russe fusillée à Sverdlovsk, des officiers blancs, des membres des partis russes non bolcheviques, des représentants des métiers libéraux qui ont souffert des mesures prises par le nouveau gouvernement (journalistes, avocats, hommes d'affaires, banquiers, etc.).

En 1923, Lénine signe un décret qui bannit des intellectuels russes et leurs familles de Russie, dont une grande partie s'installe en France. Plusieurs illustres artistes et écrivains russes trouvent ici une deuxième maison, comme les écrivains Ivan Bounine et Vladimir Nabokov, les poètes Marina Tsvetaïeva et Igor Severianine, le musicien Serguei Rachmaninov, le chanteur Fedor Chaliapine, le philosophe Nikolaï Berdiaev et tant d'autres. Le sort de beaucoup d'entre eux est loin
Paris a une image particulière dans les représentations russes, presque mythique. L'image d'une ville libre et romantique, un haut lieu de la gastronomie et de l'art de vivre à la française, la quintessence d'une vie différente et meilleure. d'avoir été facile ; en outre, l'émigration était déchirée en son sein par des clivages religieux et politiques.

Enfin, des années 1970 date une autre vague d'émigration politique russe en France. Elle se compose de dissidents expulsés par les autorités soviétiques et de transfuges qui ont choisi de ne pas rentrer lors de leur voyage ou mission en Occident. D’une manière générale, la France a attiré beaucoup de représentants de l'élite intellectuelle, littéraire et artistique russe qui ont contribué à l'enrichissement de sa culture. Un point commun réunit les représentants de toutes les vagues d'émigration : la France a toujours incarné pour eux la liberté d'opinion et d'expression que leur terre natale leur refusait.

H\&M : À la fin des années 1980, avec la dissolution de l'Union soviétique, avez-vous observé une relance de cette immigration?

T K.-J. : En effet, quand le rideau de fer disparaitt, une nouvelle vague de départs a lieu depuis la Russie vers les pays développés. Elle est différente des vagues précédentes. Les raisons qui poussent au départ sont plutôt économiques et sociales et il s'agit de décisions individuelles marquées par une grande diversité de situations. Les départs ne sont plus forcément définitifs, on assiste à une migration temporaire. Les Russes cherchent de meilleures conditions de vie. Des jeunes partent pour faire des études. Des couples mixtes se créent : il y en aurait plus de 12000 aujourd'hui. Les contacts avec les immigrés des vagues précédentes ne sont pas toujours faciles : l'émigration n'efface pas les clivages idéologiques, générationnels et de milieu social.

Les deux guerres de Tchétchénie ont augmenté le nombre de demandes d'asile politique en Europe par les ressortissants originaires du Caucase du Nord. En 2013, plus de 3000 dossiers de demandeurs d'asile sont traités en France, qui semble ainsi continuer la tradition de terre d'accueil politique pour les ex-Soviétiques. Tout récemment, le durcissement du régime de Vladimir Poutine a poussé au départ vers la France quelques personnalités comme Serguei Gouriev, économiste connu et ancien recteur d'une grande école russe.

Il est finalement difficile de connaître le nombre exact de Russes vivant en France. Tous ne sont pas enregistrés auprès du consulat et une grande partie est naturalisée. La communauté russe à Paris est généralement évaluée à 30000 personnes. En réalité, elle est peut-être bien plus grande.

\section{H\&M : En quoi Paris est une ville attractive pour les Russes parmi les autres capitales européennes?}

T K.-J. : Paris a une image particulière dans les représentations russes, presque mythique. L'image d'une ville libre et romantique, un haut lieu de la gastronomie et de l'art de vivre à la française, la quintessence d'une vie différente et meilleure. Cette image a été cultivée à travers la littérature, la poésie et le cinéma russe et soviétique. D’ailleurs, les attentes peuvent être tellement grandes que Paris peut décevoir une fois qu'on y est ! L'une de 
mes anciennes étudiantes l'a formulé un jour ainsi : "Je m'attendais à voir un Parijichtché (Grand Paris), mais en vérité ce n'est qu'un Parijek (Petit Paris)." Blasés par les voyages aux services impeccables vers les destinations exotiques, certains Russes font la fine bouche en évoquant Paris, mais continuent à y revenir, à cause du charme des terrasses parisiennes ou en quête de bonnes affaires dans les boutiques à la mode. Ceux qui s'installent à Paris découvrent progressivement ce Parijek de proximité, où les commerçants se souviennent de leurs habitudes.

$H \& M$ : Quels sont les quartiers de résidence des Russes à Paris? Ces quartiers ont-ils donné lieu à un développement de commerces russes spécialisés (traiteurs, libraires, tourisme, etc.) ?

T K.-J. : Historiquement, il y avait une concentration des Russes dans le XVe arrondissement, plus populaire, et le XVIe, plus nanti. C'est dans ces quartiers qu'on retrouve la plupart des lieux de mémoire liés à l'émigration russe ou des églises orthodoxes, à l'exception notable de la cathédrale de la rue Daru. Mais il y a eu aussi beaucoup de Russes dans le XVII ou à Boulogne-Billancourt, où se trouvaient des usines Renault. Il semblerait que les Russes y étaient si nombreux après la Révolution de 1917 qu'on l'appelait Billankoursk!

Aujourd'hui, la diaspora est bien plus dispersée à Paris et dans les banlieues. Quasiment dans tous les arrondissements de Paris, on peut trouver une ou plusieurs épiceries russes, dont la plupart des produits vendus viennent... d'Allemagne, où la diaspora russe est très nombreuse et active. Les boutiques de souvenirs, les cafés et les restaurants russes sont nombreux à Paris. Un centre de divertissement "Village russe" dans le XIII arrondissement cherche à symboliser l'art de vivre "à la russe". Les deux librairies russes les plus connues - la Librairie du Globe dans le III et les Éditeurs réunis dans le $\mathrm{V}^{\mathrm{e}}$ - subissent pleinement la crise du livre. Celle du Globe, qui existe depuis 1952, a failli fermer il y a deux ans et a été sauvée in extremis par un don de Youri Kovaltchouk, homme d'affaires proche de Vladimir Poutine. Les deux librairies diversifient leurs activités en organisant des événements autour de livres ou d'auteurs.

\section{$H \& M$ : Quels sont les secteurs économiques qui attirent les investissements des ressortissants russes à Paris ?}

T K.-J. : Il y aurait aujourd'hui une quarantaine de projets d'investissements en France liés au capital russe. Il n'est pas toujours évident de distinguer les investissements des Russes qui vivent à Paris de ceux qui investissent depuis la Russie. Certains investissements ne sont probablement pas comptabilisés, venant des offshores ou via un autre pays européen. Quoi qu'il en
Quasiment dans tous les arrondissements de Paris, on peut trouver une ou plusieurs épiceries russes, dont la plupart des produits vendus viennent... d'Allemagne, où la diaspora russe est très nombreuse et active. soit, la France est loin d'être le premier pays pour les investissements russes en Europe, même si, ces dernières années, la dynamique a été plutôt positive. Les Russes semblent rencontrer plusieurs difficultés pour investir ici : l’image dégradée de leur pays ne facilite pas l'ouverture des comptes ou l'accès aux crédits bancaires. La vigilance vis-à-vis de la provenance de l'argent russe est de mise. Certains Russes vont jusqu'à dire qu'ils n'investissent en France que dans les secteurs qui n'existent pas ailleurs, notamment la production de vin, de cognac et de champagne. En effet, plus de la moitié des projets russes en France sont liés à l'agroalimentaire, hors Paris. On compte aussi en dehors de Paris quelques grands projets industriels dans le matériel aéronautique, naval et ferroviaire, ainsi que des laboratoires de recherche et développement.

La région parisienne accueille environ un tiers des investissements russes en France. Dans le Grand Paris, il faut mentionner la construction de deux tours jumelles "Hermitage Plaza" à La Défense : de 323 mètres chacune, elles devront accueillir 
dans quelques années des bureaux, des commerces, une galerie d'art, une salle de concert, un hôtel, un lycée russe et une résidence universitaire. Le chantier est mené par le groupe de l'entrepreneur russe installé à Paris Emin Iskanderov. Cette présence russe très remarquée reste cependant exceptionnelle parmi les promoteurs immobiliers à Paris.

Certaines enseignes françaises ont été rachetées par des Russes, comme Hédiard par l'ancien banquier Sergueï Pougatchev en 2007. Cependant, ce projet - qui a d'ailleurs fait faillite fin 2013 - n'a pas cherché à mettre en avant l'identité russe, à la différence du Café Pouchkine du Printemps Haussmann. Il y a aussi des investissements assez inattendus : le groupe OGF, le leader français des pompes funèbres, a été racheté par une société liée au groupe Alfa de l'oligarque

Il y a, à Paris, quelques véritables trésors russes cachés. Par exemple, des isbas datant de l'Exposition universelle de 1867 et toujours habitées (!) se cachent dans une impasse à Ranelagh.

écoles associatives du mercredi existent désormais à Paris avec des méthodes d'enseignement russes pour les jeunes bilingues. Cours de langue, de musique ou de peinture, services juridiques, traductions, accompagnement de touristes, organisation de concerts, de tournées et de soirées thématiques - les Russes de Paris excellent dans beaucoup de métiers d'art et d'esprit.

\section{$H \& M$ : Peut-on imaginer que ces investisse- ments vont susciter une plus forte visibilité de la culture russe à Paris?}

T K.-J. : Compte tenu des secteurs d'investissements que j'ai décrits, pas tellement, sauf peutêtre la future galerie d'art à La Défense. Il y a eu au milieu des années 2000 l'initiative du banquier Alexandre Lebedev d'ouvrir un centre culturel "Château des Forgets" à une heure de route de
Paris. Le lancement de ce projet s'est fait en grande pompe, mais finalement, très élitiste, il est inconnu de la diaspora. D'ailleurs, on ne sait même pas s'il existe encore.

La visibilité de la culture russe est bien assurée par les tournées des troupes venant de Russie ou des événements annuels comme les festivals de cinéma ou de musique. Les Journées du livre russe avec la remise du prix Russophonie pour les meilleures traductions du russe vers le français, des événements qui sont très attendus et ont maintenant leurs habitués. Les ballets russes ou les concerts de Valery Gergiev font toujours salle comble. Le Centre culturel et scientifique russe organise régulièrement des manifestations culturelles. En 2012, l’année croisée France-Russie a rendu honneur à la culture russe en France. Plusieurs associations à vocation culturelle contribuent à la richesse de la vie "à la russe" dans la capitale française.

Il faut aussi mentionner une absence notable dans les investissements : il n'y a pas de "Maison russe" à la Cité universitaire de Paris. Pourtant, il en est question depuis plusieurs années. Les universités et les grandes écoles françaises ont accueilli l'année dernière plus de 5000 étudiants russes et la France est le $4^{\mathrm{e}}$ pays d'accueil pour les étudiants russes en mobilité internationale. Le besoin de déléguer la gestion de la propriété à la Cité universitaire fait fuir les investisseurs russes potentiels, qui semblent encore avoir du mal avec le concept de mécénat dans ce domaine.

H\&M : Quels sont les monuments historiques qui symbolisent le patrimoine de cette immigration russe à Paris ? Ces bâtiments ont-ils encore un usage dans la communauté russe ?

T K.-J. : Sans parler des bâtiments officiels comme l'ambassade boulevard Lannes, la résidence de l'ambassadeur rue de Grenelle, ou le Centre culturel et scientifique rue Boissière, qui ont tous leur propre histoire, plusieurs monuments symbolisent la présence historique russe à Paris. Certains sont bien visibles, connus et fréquentés. La plupart sont liés 
à l'époque impériale ou à la vague d'émigrés blancs : la cathédrale de la rue Daru, le musée Zadkine, le conservatoire Rachmaninov. Aux environs de Paris, il y a des lieux de mémoire très importants pour les Russes comme le cimetière de Saint-Genevièvedes-Bois ou le musée Tourgueniev à Bougival.

Certains ont perdu avec le temps leur valeur symbolique et sont tombés dans l'oubli. C'est le cas du musée Lénine (rue Marie-Rose dans le XVI') et de la Ruche (passage Dantzig, Paris XVe), cette cité d'artistes pour les peintres et sculpteurs désargentés du début du XX siècle. Cette dernière a pour- tant accueilli plusieurs illustres artistes russes, dont Marc Chagall, auteur du plafond de l'Opéra de Paris. Quelques monuments ont été érigés tout récemment, comme celui au corps expéditionnaire russe ${ }^{1}$ inauguré en 2010 à côté du pont Alexandre-III. Mais il y a aussi à Paris quelques véritables trésors russes cachés. Par exemple, des isbas, datant de l'Exposition universelle de 1867 et toujours habitées (!) se cachent dans une impasse à Ranelagh. Une petite merveille, une église orthodoxe à la coupole bleue, se trouve derrière une grande porte cochère rue Lecourbe dans le XVe. On ne la verra pas si on ne sait pas où la chercher ${ }^{2}$ ! 
$H \& M$ : Les médias en langue russe sont-ils nombreux à Paris ? Sont-ils un facteur dynamique pour créer du lien au sein de cette communauté ? T K.-J. : Il existe quelques journaux et revues en russe à Paris. La plus ancienne est Rousskaïa Mysl (La Pensée russe), créée en 1947, qui d'ailleurs depuis quelques années n'est plus éditée à Paris, mais à Londres. Le Centre de langue

Les orthodoxes de Paris

estiment pour la plupart

qu'il y a un besoin rée

d'une nouvelle cathédrale, les petites églises des quartiers

étant pleines à craquer le dimanche. et de culture russe publie depuis 1987 La Gazette tous les deux mois, qui contient des actualités, mais aussi des petites annonces. Un bulletin d'annonces russes à Paris, Sovetnik (Conseiller) est diffusé gratuitement en version papier et en version numérique. En radio, il existe depuis 2006 Capitales Radio, destinée au public russophone, ainsi que des émissions de Russkij otchevidetz (L'Observateur russe), site d'information franco-russe basé à Paris depuis 2009. Cependant, les deux sont très peu connus de la diaspora. Il n'existe pas de chaîne de télévision qui lui soit dédiée. La plupart des Russes sont à la fois bien intégrés dans la société française et maîtrisent le français, ils ont accès aux médias russes via Internet ou le bouquet satellitaire. La diffusion de l'actualité des Russes en France n'est donc pas tellement assurée par les médias classiques, mais par une constellation de sites, portails, bulletins et lettres d'information. C'est un lien vivant et instantané entre les membres de la communauté.

H\&M : En 2016, une cathédrale orthodoxe sera inaugurée à deux pas de la tour Eiffel. Elle pourra accueillir une communauté religieuse vaste à Paris, estimée à 200000 pratiquants. Depuis combien d'années ce projet est-il sur les rails ? En quoi est-il significatif de cette histoire des Russes avec Paris?

T K.-J. : Le projet comprend une cathédrale, un centre paroissial, une école bilingue et un centre culturel. Il a été validé en 2007 par Nicolas Sarkozy lors de la visite du précédent patriarche de Moscou, Alexis II. Trois ans plus tard, l'État russe a acquis le terrain de l'ancien siège de Météo France quai Branly pour 70 millions d'euros.

Les orthodoxes de Paris estiment pour la plupart qu'il y a un besoin réel d'une nouvelle cathédrale, les petites églises des quartiers étant pleines à craquer le dimanche. En même temps, cette construction changera l'équilibre existant depuis des années à Paris entre les paroisses relevant du Patriarcat de Moscou et de celui de Constantinople. Les relations entre les deux branches orthodoxes, liées à l'histoire de l'émigration blanche, ont toujours été tendues, certaines églises orthodoxes russes à l'étranger rejetant l'autorité de Moscou pendant toute la période soviétique. Même si les relations semblent apaisées aujourd'hui, avec ce projet, la plus grande cathédrale à Paris ne relèvera plus de Constantinople, comme c'est le cas avec Saint-Alexandre-Nevsky de la rue Daru, mais de Moscou. En connaissant la montée de l'influence de la religion en Russie postsoviétique, il n'est pas étonnant que l'Église orthodoxe russe cherche à affirmer sa puissance retrouvée et à rayonner sur ce qu'on appelle le Rousskij mir (monde russe) au-delà des frontières nationales. La proximité entre les autorités politiques russes et l'Église orthodoxe fait que ce projet relève aussi du soft power de l'État russe. Le fait que l'État le finance en est la preuve.

H\&M : Ce projet fortement appuyé par Poutine a suscité un débat polémique. Pourquoi ? Quels étaient les détracteurs parisiens de ce projet? À quel retentissement faut-il s'attendre à cette occasion?

T K.-J. : Le projet n'était pas du goût de tout le monde. Pourtant, un concours international a été organisé : dix propositions finales ont circulé et la diaspora russe a pu exprimer son choix. Le projet final comprenait une église surmontée de cinq bulbes dorés, dont le plus haut devait atteindre une trentaine de mètres, l'ensemble recouvert d'un voile en verre. L'ancien maire de Paris, Bertrand Delanoë, 
a été le détracteur le plus virulent du projet. Sans remettre en cause le principe d'une église orthodoxe sur ce site, il dénonçait la médiocrité du projet "conçu dans la précipitation", une "architecture de pastiche" qui relevait d'une "ostentation inadaptée au site classé au Patrimoine mondial de l'Unesco ou à la perspective de la tour Eiffel". La Mairie de Paris ne disposait que d'une voix consultative sur ce dossier. Cependant, le projet a été repris de zéro et l'architecte lauréat du concours a été écarté : avant de naître, la cathédrale fait donc l'objet d'un procès. Le nouveau projet ne bénéficie pas d'une communication active : il semblerait qu'il s'inspire de la cathédrale de la Dormition de Moscou, la première église en pierre et la plus ancienne du Kremlin, où les tsars étaient couronnés. Depuis le changement de projet, aucune objection n'a été formulée par la Mairie de Paris.

Le projet a démarré en 2014 et le chantier devrait se terminer dans deux ans. Compte tenu de la dégradation des relations entre la Russie et l'Europe et de l'image de la Russie à la suite de la crise en Ukraine, ainsi que de l'intérêt personnel de Vladimir Poutine pour ce projet, il n'est pas à exclure que ce dossier revienne d'une manière ou d'une autre au centre des relations franco-russes. 